\title{
App-assured essential physical activity for the prevention of cognitive decline: changing paradigms in public health - a study protocol for a randomised controlled trial
}

\section{Małgorzata Jamka}

Department of Pediatric Gastroenterology and Metabolic Diseases, Poznan University of Medical Sciences, Poznań, Poland

(iD) https://orcid.org/0000-0002-0257-6180

\section{Aleksandra Makarewicz}

Department of Pediatric Gastroenterology and Metabolic Diseases, Poznan University of Medical Sciences, Poznań, Poland

(iD) https://orcid.org/0000-0001-9310-9643

\section{Maria Wasiewicz-Gajdzis}

Department of Pediatric Gastroenterology and Metabolic Diseases, Poznan University of Medical Sciences, Poznań, Poland

(iD) https://orcid.org/0000-0002-7084-663X

\section{Jan Brylak}

Department of Pediatric Gastroenterology and Metabolic Diseases, Poznan University of Medical Sciences, Poznań, Poland

(iD) https://orcid.org/0000-0003-1398-3387

\section{Hanna Wielińska-Wiśniewska}

Department of Pediatric Gastroenterology and Metabolic Diseases, Poznan University of Medical Sciences, Poznań, Poland

(iD) https://orcid.org/0000-0001-5242-1059

\section{Zuzanna Pawlak}

Department of Pediatric Gastroenterology and Metabolic Diseases, Poznan University of Medical Sciences, Poznań, Poland

(iD) https://orcid.org/0000-0002-1947-1481

\section{Jan Krzysztof Nowak}

Department of Pediatric Gastroenterology and Metabolic Diseases, Poznan University of Medical Sciences, Poznań, Poland

(D) https://orcid.org/0000-0003-0953-2188

\section{Karl-Heinz Herzig}

Department of Pediatric Gastroenterology and Metabolic Diseases, Poznan University of Medical

\section{Sciences, Poznań, Poland \& Research Unit of} Biomedicine, University of Oulu, Oulu, Finland

(iD) https://orcid.org/0000-0003-4460-2604

\section{Edyta Mądry}

Department of Physiology, Poznan University of Medical Sciences, Poznań, Poland

(iD) https://orcid.org/0000-0002-0081-6558

\section{Jarosław Walkowiak}

Department of Pediatric Gastroenterology and Metabolic Diseases, Poznan University of Medical Sciences, Poznań, Poland

(iD) https://orcid.org/0000-0001-5813-5707

Corresponding author: jarwalk@ump.edu.pl

DOI: https://doi.org/10.20883/medical.e530

Keywords: physical activity, cognitive functions, mild cognitive impairment

Published: 2021-09-01

\begin{abstract}
How to Cite: Jamka M, Makarewicz A, Wasiewicz-Gajdzis M Brylak J, Wielińska-Wiśniewska H, Pawlak Z, Nowak JK, Herzig K-H, Mądry E, Walkowiak J. App-assured essential physical activity for the prevention of cognitive decline: changing paradigms in public health - a study protocol for a randomised controlled trial: A study protocol of the PA PROTECT study. Journal of Medical Science. 2129;90(4):e530. doi:10.20883/medical.e530
\end{abstract}

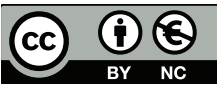

C 2021 by the author(s). This is an open access article distributed under the terms and conditions of the Creative Commons Attribution (CC BY-NC) licencse. Published by Poznan University of Medical Sciences

\section{ABSTRACT}

This study aims to assess the effect of an increase in daily physical activity to prevent cognitive decline, sustain brain volumes and maintain healthy biomarker levels in mild cognitive impairment (MCl) subjects 
aged 50-70 years. In total, 198 subjects with $\mathrm{MCl}$ (assessed using the Montreal Cognitive Assessment test) will be recruited and randomised into two groups: active and passive. The active group will be instructed, encouraged and motivated to increase their physical activity to at least a moderate level ( $\geq 10,000$ steps/day), whereas the passive group should maintain their normal activity levels. All subjects will undergo cognitive assessment, neuroimaging and biomarker tests prior to and after a one-year intervention. During the intervention, physical activity will be measured by the Fitbit Inspire HR wristband. The study was registered in the German Clinical Trials Register database (registration no. DRKS00020943, date of registration: 09.03.2020, protocol version: 1.0).

\section{Research Project Objectives}

The study aims to assess the effect of increasing daily physical activity on the prevention of cognitive decline, sustaining brain volumes, as well as on maintaining healthy biomarker levels in subjects aged $50-70$ years affected by mild cognitive impairment $(\mathrm{MCl})$. The study hypotheses are as follows:

1. Increased daily physical activity to at least a moderate level ( $>10,000$ steps/day) for one year will not affect cognitive function.

2. Higher levels of daily physical activity (> 10,000 steps/day) will not preserve brain volume and will not maintain proper values of healthy biochemical markers and anthropometric parameters.

\section{Research Plan and Basic Concept}

\section{Basic Concept}

$\mathrm{MCl}$ is a condition in which subjects demonstrate cognitive decline with minimal dysfunction of instrumental daily activities, which may also be a stage preceding dementia [1]. According to a recent systematic review, about $18 \%$ of $\mathrm{MCl}$ subjects develop dementia within two years, with the conversion rate increasing to $32 \%$ following five years [2]. In 2016, the global prevalence of dementia was 48.3 million [3], which is anticipated to increase to 80 million in 2030 [4]. Neurocognitive disorders significantly affect everyday living and place a substantial financial burden on healthcare systems. Advanced age and family history of neurocognitive disorders are important risk factors for developing dementia, as well as numerous modifiable risk factors, such as hypercholesterolaemia, hypertension, obesity, hyperglycaemia, poor education and physical inactivity
[5]. Moreover, currently, there is no pharmacological treatment approved for $\mathrm{MCl}$. Therefore, it is crucial to identify $\mathrm{MCl}$ subjects and attempt to mitigate the risk factors in this group [6].

To date, there have been several studies regarding the impact of physical activity on the prevention of cognitive decline [7-9]. A recent meta-analysis demonstrated that physical activity (aerobic, resistance training or tai chi) positively affects cognitive function in adults aged 50 years or older, regardless of their baseline cognitive status [7]. In addition, another meta-analysis showed that slow walking and jogging significantly improved attention, execution and memory processes [8]. Furthermore, improved daily physical activity, defined as walking a greater distance, helps to preserve grey matter volume in the frontal, occipital, entorhinal, and hippocampal regions, resulting in a reduced risk of cognitive decline $[9,10]$.

Although several studies reported a relationship between physical activity and cognitive functions preservation, there is no consensus regarding the exact frequency, duration, intensity and type of exercise necessary to prevent cognitive decline. Current physical activity guidelines recommend that adults should be involved in at least 150 minutes of moderate-intensity aerobic exercise a week $[4,11]$. However, only less than $5 \%$ of adults were able to comply with the recommendations which indicates that the existing guidelines are too demanding for the elderly. Moreover, actual physical activity was lower compared to the declared level of activity in all forms of questionnaires [12].

\section{Study design}

The study was designed as a parallel-group prospective randomised controlled trial. The study protocol was registered in the German Clini- 
cal Trials Register database (registration no. DRKS00020943, date of registration: 09.03.2020, protocol version: 1.0). The study protocol is reported in accordance with the Standard Protocol Items: Recommendations for Interventional Trials guidelines $[13,14]$. The data in this study will be reported and presented according to the Consolidated Standards of Reporting Trials statement [15]

\section{Ethical issues}

The present study will be conducted according to the guidelines provided in the Declaration of Helsinki. The study protocol was approved by the Bioethics Committee of Poznan University of Medical Sciences (refs. 47/20, 169/20, 263/20, $481 / 20,720 / 20,296 / 21$ and 555/21). The study personnel will obtain written informed consent from all study participants upon their enrolment. The study will not have a data monitoring committee, given that we do not anticipate severe adverse effects. Study-related personal damage of the participating subjects will be covered by the Poznan University of Medical Sciences insurance policy. Should the study protocol require amendments, a revised version will be submitted to the Bioethics Committee. The study team and the study participants will be also informed about all the changes.

\section{Study population}

In total, 198 subjects will be recruited to the study. Inclusion criteria are as follows: age 50-70 years, diagnosed $\mathrm{MCl}$ (the result of the Montreal Cognitive Assessment (MoCA) test: 19-26 points), community residence, and owning a smartphone. On the other hand, the exclusion criteria include: depression and/or the results of the Hamilton Depression Rating Scale (HAM-D) $>13$ points, use of cognitive boosting medications or psychotropic medications, substance abuse disorders (e.g. alcohol > 15 drinks (units)/ week), diagnosed psychiatric disorders, Parkinson's disease, Alzheimer's disease, dementia, anaemia, diabetes of at least 10 years, chronic renal and liver diseases, a history of cancer within the past five years, history of stroke, current evidence or a history of seizures in the past two years, head injury with loss of consciousness and/or immediate confusion following the injury, hypothyroidism with current misaligned thyrotrophic hormone levels, any chronic diseases which limit training and testing of cardiovascular and respiratory systems, current intensive physical activity (at least 10,000 steps/ day), implanted pacemaker, neurostimulator and other metal components, including prosthetic implants, blindness, deafness, language difficulties or any other disability which may prevent subjects from participating, or cooperating in the protocol.

\section{Recruitment}

Participants will be recruited to the study from patients of medical clinics and medical centres in the Greater Poland region (Poland) in consultation with their physicians and directors of the clinics, by means of study promotion via workplace channels at the university and healthcare services, as well as via university newsletters and websites, posters, leaflets and email invitations sent to companies, offices, and institutions for distribution to their employees. The research team will contact the interested participants and send further information about the study. Prior to the commencement of the study, the potential subjects will be screened by a physician during an inclusion appointment to comply with the protocol requirements. In this phase, cognitive functions will be evaluated by the MoCA test and the HAM-D scale will be used to assess the occurrence of depression symptoms. Additionally, physical activity will be determined for at least one week before the enrolment using the Fitbit Inspire HR tracker. Subjects will receive information regarding the study, its purpose, putative benefits, and the possible risks. All subjects will be informed that participation in that study is voluntary, and that they may refuse to participate, or withdraw from the trial at any time without providing reasons.

\section{Intervention}

The study population will be randomised (allocation ratio: 1:1) into two groups: active (group $A$ ) or passive (group $P$ ). Group $P(n=99)$ will be asked to sustain their normal activity, whereas group A $(n=99)$ will be asked, instructed and motivated by the mobile application to increase their physical activity intensity to at least 10,000 steps/day. During the intervention period, all subjects will be instructed to maintain their current diet and 
maintain their medications and, if they change, to record this in a diary. Prior to and after the one-year intervention period, cognitive functions, neuroimaging, and biochemical parameters will be assessed in all study subjects. Additionally, physical activity will be determined using the Fitbit Inspire HR tracker. Moreover, before, during and after the intervention period, anthropometric and densitometric parameters, body composition, as well as dietary habits will be assessed. A self-administered questionnaire regarding physical activity, health condition, medications, smoking, alcohol use, profession, and educa- tion will be distributed to subjects. Basic clinical examinations and measurements will also be performed. The scheme of this study is presented in Figure 1.

\section{Adherence to the intervention}

Adherence to the intervention will be assessed by data collected from the Fitbit, including the number of steps per day, distance travelled, estimated energy expenditure, sedentary behaviour, minutes of low, moderate and intensive activity, as well as sleep behaviour. The data will be wirelessly uploaded to the user's account and will

\begin{tabular}{|c|c|c|c|c|}
\hline & \multicolumn{4}{|c|}{ STUDY PERIOD } \\
\hline & Enrolment & Allocation & Post-allocation & Close-out \\
\hline TIMEPOINT & $-t_{1}$ & $t_{0}$ & $\boldsymbol{t}_{\boldsymbol{l}}\left(6^{\text {th }}\right.$ month $)$ & $\boldsymbol{t}_{2}\left(12^{\text {th }}\right.$ month $)$ \\
\hline ENROLMENT: & & & & \\
\hline Eligibility screen & $\mathrm{x}$ & & & \\
\hline Informed consent & $\mathrm{x}$ & & & \\
\hline Medical examination & $\mathrm{X}$ & & & \\
\hline $\begin{array}{r}\text { Hamilton Depression Rating } \\
\text { Scale }\end{array}$ & $\mathrm{x}$ & & & \\
\hline Montreal Cognitive Assessment & $\mathrm{x}$ & & & \\
\hline $\begin{array}{r}\text { Physical activity } \\
\text { (Fitbit) }\end{array}$ & $\mathrm{x}$ & & & \\
\hline Allocation & & $\mathrm{X}$ & & \\
\hline INTERVENTIONS: & & & & \\
\hline $\begin{array}{r}\text { Active group } \\
(>10000 \text { steps/day) }\end{array}$ & & & & \\
\hline $\begin{array}{r}\text { Passive group } \\
(<10000 \text { steps/day })\end{array}$ & & & & \\
\hline ASSESSMENTS: & & & & \\
\hline Primary outcomes: & & & & \\
\hline Montreal Cognitive Assessment & $\mathrm{x}$ & & & $\mathrm{x}$ \\
\hline $\begin{array}{r}\text { Cambridge Neuropsychological Test } \\
\text { Automated Battery }\end{array}$ & & $\mathrm{X}$ & & $\mathrm{X}$ \\
\hline Secondary outcomes: & & & & \\
\hline $\begin{array}{r}\text { Anthropometric parameters (body high, body } \\
\text { weight, waist and hip circumferences, body } \\
\text { mass index) }\end{array}$ & & $\mathrm{X}$ & & $\mathrm{x}$ \\
\hline $\begin{array}{r}\text { Biochemical markers } \\
\text { (fasting glucose and insulin homeostasis } \\
\text { markers, lipid profile, inflammatory markers, } \\
\text { markers of neuronal growth and destruction) }\end{array}$ & & $\mathrm{X}$ & & $\mathrm{X}$ \\
\hline Blood pressure & & $\mathrm{X}$ & & $\mathrm{X}$ \\
\hline $\begin{array}{r}\text { Body composition and densitometric } \\
\text { parameters (DEXA) }\end{array}$ & & $\mathrm{X}$ & & $\mathrm{x}$ \\
\hline Food Frequency Questionnaire & & $\mathrm{x}$ & $\mathrm{x}$ & \\
\hline $\begin{array}{r}\text { Hamilton Depression Rating } \\
\text { Scale }\end{array}$ & $\mathrm{X}$ & & & $\mathrm{X}$ \\
\hline International Physical Activity Questionnaire & & $\mathrm{x}$ & $\mathrm{X}$ & $\mathrm{X}$ \\
\hline Magnetic resonance imaging & & $\mathrm{x}$ & & $\mathrm{x}$ \\
\hline $\begin{array}{r}\text { Physical activity } \\
\text { (Fitbit) }\end{array}$ & & $\mathrm{X}$ & $\mathrm{X}$ & $\mathrm{X}$ \\
\hline Socioeconomic assessment & & $\mathrm{x}$ & & $\mathrm{x}$ \\
\hline 3-day dietary record & & $\mathrm{X}$ & & $\mathrm{x}$ \\
\hline
\end{tabular}

Figure 1. The study schedule of the enrolment, interventions, and assessments 
be downloaded by our research team through the Fitbit website, or by means of the application programming interface. The Fitbit data will provide us with objective information regarding the level of adherence throughout the intervention period. Furthermore, in order to increase adherence to the intervention, phone calls will be scheduled to review the compliance with the physical activity guidelines, and all participants will also be given the option of additional calls if necessary. In addition, weekly emails, including the information about their average step count, will be sent to the study participants. Moreover, a check-up appointment will be conducted six months after starting the intervention in order to verify the subject's adherence to the intervention. The study participants who will not comply with the intervention will be excluded from the study, and the principal investigator will make a final decision regarding the exclusion. If a participant decides to withdraw from the study, no further data will be collected concerning this individual.

\section{Minimum sample size calculation}

The minimum sample size was calculated on the basis of a recent physical activity intervention study in subjects with an incident of cognitive impairment. It demonstrated that the executive function and memory scores were -0.33 \pm 0.79 and $-0.32 \pm 1.29$, in the low active and $0.31 \pm 0.86$, and $0.22 \pm 1.05$ in the highly active group [16]. However, we assume that due to the preselection, we will manage to obtain a more homogenous group, and thus achieve a more significant clinical effect and 1) the probability of a type-I error at an alpha cut-off level of $5 \%$ $(\alpha=0.05) ; 2)$ the probability of a type-II error at a beta cut-off level of $20 \%(\beta=0.2) ; 3)$ the difference of the anticipated means equals to 0.62 standard deviation (SD); 4) the expected value of SD equals to $85 \%$ of the mean.

\section{Randomisation and blinding}

Randomisation will be performed via computer software (RRApp Robust Randomization App, the Icahn School of Medicine at Mount Sinai, New York, NY, USA [17]) and the data will be uploaded by an independent researcher. We will perform blocked randomisation (block size: six) with the stratification according to sex and prevalence of diabetes. The participants will be allocated in equal numbers to one of two groups, passive or active, as defined by the code. According to the character of the intervention, the study participants and researchers taking the physical activity measurements will not be blinded to the allocation. Only the outcome assessors and the study team members who will prepare the database and will perform the statistical analysis will be blinded.

\section{Protection of data privacy}

Quantitative data will be collected from the recruited subjects using anthropometric, clinical, biochemical and behavioural measurements to written and electronic files, and subsequently to permanent file formats for analysis. Subjects will be identified by non-personal codes and tied to metafiles. The data will be verified by investigators. Original written documents will be stored in a locked filing cabinet, whereas all the data will be collected in secure access computers. Documents and files will be retained as authorised by the Bioethics Committee. The final trial dataset will be accessed by the principal investigator, study coordinator and other team members.

\section{Dissemination}

The study results will be presented at local, national and international conferences, and will be published in open-access peer-reviewed journals. Authorship eligibility will be based on the International Committee of Medical Journal Editors. The data collected in this study will be available on request from the principal investigator. Study participants will be informed of the outcomes of the study.

\section{Research Methodology}

\section{Primary and secondary outcomes}

The primary outcomes of the study will be changes ( $\Delta$ before - after) in cognitive function parameters assessed by the MoCA test and the Cambridge Neuropsychological Test Automated Battery (CANTAB), whereas the changes in biochemical parameters, neuroimaging, anthropometric parameters, body compositions and densitometric parameters will be regarded as the secondary outcomes. All the data, except neuroimaging, will 
be collected in the Department of Pediatric Gastroenterology and Metabolic Diseases, Poznan University of Medical Sciences. Brain magnetic resonance imaging (MRI) will be performed at the Heliodor Swiecicki Clinical Hospital in Poznan. Blood samples will be collected by a commercial laboratory, while the biochemical parameters will be measured at the Laboratory of the Department of Pediatric Gastroenterology and Metabolic Diseases, Poznan University of Medical Sciences, or by a commercial laboratory.

\section{Anthropometric measurements}

Basic anthropometric parameters (body height, body weight, hip and waist circumferences) will be measured before and after the intervention period. Body mass index will be calculated on the basis of body weight and body height measurements. During the anthropometric measurements, all participants will wear light clothes and will be barefoot, with an average of two measurements recorded.

Body composition and densitometric parameters Before and after the intervention period, body composition (fat and free fat mass), bone mineral density and the content of the total body and lumbar spine (L1-L4) will be assessed by means of dual-energy X-ray absorptiometry methods using the Hologic Discovery analyser (Bedford, Massachusetts, USA).

\section{Blood pressure}

Blood pressure will be measured prior to blood sample collection according to the guidelines of the European Society of Hypertension. Blood pressure will be measured on the arm at the heart level, and will be expressed by three measurements of the systolic and diastolic pressure [18].

\section{Assessment of dietary habits}

Dietary habits will be assessed before and during the intervention period by means of 3-day dietary records covering two weekdays and one weekend day. Participants will be asked to give a detailed description of foods consumed and to estimate their quantity. To investigate food group intake, the Beliefs and Eating Habits Questionnaire created by the Behavioural Conditions of Nutrition Team, Committee of Human Nutrition Science of the Polish Academy of Sciences will be adminis- tered [19]. The energy intake and the basic nutritional compounds (carbohydrates, proteins, and fats), selected vitamins and minerals, dietary fibre, cholesterol, saturated, monounsaturated and polyunsaturated fatty acids intake will be assessed using the Aliant software (Anmarsoft, Gdańsk, Poland). The nutrition standards for the Polish population will be applied to determine whether individual dietary intakes meet the nutritional recommendations [20].

\section{Physical activity}

Physical activity will be determined using the Fitbit Inspire HR tracker (Fitbit Inc., San Francisco, USA). In addition, the International Physical Activity Questionnaire will be used to assess physical activity before, during and after the intervention period.

Fitbit Inspire HR is a wrist-worn wearable wireless sensor with an accelerometer recording physical activity throughout the day, which can synchronise with a smartphone application and a computer. Therefore, participants will be instructed to download the Fitbit app, and will be asked to wear the Fitbit all day, except when showering, bathing, and swimming. Participants will be instructed to wear the Fitbit on their non-dominant wrist for one year. In general, the Fitbit requires the creation of individual user accounts to download the stored data using a Web-based software application. Nevertheless, for the purpose of this study, user accounts will be created by the study team which can only be accessed by the researchers. Physical activity data will be stored on the individual accounts of study participants and will be downloaded of each participant's wearing period by the study team.

\section{Hamilton depression rating scale}

The HAM-D scale was used during the inclusion visit and after the intervention to assess the prevalence of depression symptoms [21]. The scale predominantly assesses cognitive and vegetative symptoms, with relatively few items related to social, motor, anxiety and mood factors. The 17-item HAM-D was employed in the present study, each item is scored from 0 to 2 or from 0 to 4, with total scores ranging from 0 to 52 . The following cut-off points were used: $\geq 23-$ very severe depression, 18-22 - severe depres- 
sion, 14-18 - moderate depression 8-13 - mild depression and $<7-$ not depressed [22].

\section{Cognitive assessments}

The CANTAB and MoCA tests will be employed in this study as the primary outcome parameters. The included tests comprise the following categories: executive functioning, processing speed, memory and abbreviated memory. The following test batteries will be involved in the present study: Motor Screening Task, Reaction Time, Paired Associates Learning, Spatial Working Memory, Pattern Recognition Memory, Delayed Matching to Sample, Rapid Visual Information Processing.

\section{Neuroimaging protocol}

Brain magnetic resonance imaging will be performed on all subjects using a Siemens Skyra 3T magnetic resonance imaging (MRI) System. The following MRI sequences will be used in every examination: 1) T2-weighted (fast-spin echo) and modified T2-weighted fluid-attenuated inversion recovery sequence for the detection and localisation of ischaemic lesions; 2) diffusion-weighted with an apparent diffusion coefficient map for the detection of acute ischaemic focuses; 3 ) susceptibility-weighted imaging for identification of intracerebral haemorrhagic and microhaemorrhagic lesions; 4) 3D angiographic time of flight sequence for visualisation of blood flow in intracerebral arterial vessels; 5) T1-weighted 3D spoiled gradient-recalled echo sequence (3D volumetric sequence) for estimation of total and segmented brain volume.

\section{Blood collection and biochemical analysis}

Blood samples will be collected from the antecubital vein via standard venepuncture performed by registered staff nurses. The samples will be taken from the participants after 12-h fasting. The following blood biomarkers will be measured: fasting glucose and insulin homeostasis markers, lipid profile, inflammatory markers (interleukin 6 , interleukin 1 receptor type alpha, tumour necrosis factor-alpha, high-sensitivity C-reactive protein), as well as neuronal growth and destruction markers (brain-derived neurotrophic factor, amyloid $\beta-40$, amyloid $\beta-42$ ratio and phosphorylated Tau protein). Other biochemical and genetic analyses are planned as optional if further funding is available.

\section{Sociodemographic and medical history} questionnaires

Background, place of residence, education, family status, and economic status will be assessed before and after the intervention using a sociodemographic questionnaire. The participants will also answer questions regarding lifestyle factors, including tobacco smoking habits and alcohol consumption. A medical history questionnaire will be used to assess the health status of the study participants and to verify whether the subjects receive any medications or dietary supplements.

\section{Statistical analyses}

The STATISTICA (StatSoft, Tulsa, USA) software, or equivalent, will be used for the statistical analysis. A two-sided $p$-value $<0.05$ will be considered statistically significant. The overall characteristics of subjects will be expressed as a mean and SD with $95 \%$ confidence interval, median and interquartile range, or as frequencies and percentages. The outcomes will also be expressed as changes between the postand pre-intervention values ( $\Delta$ value at 1 year). The normality of the variable distribution will be verified on the basis of the Shapiro-Wilk normality test. Comparisons between two unpaired groups will be determined using t-tests or Mann-Whitney $U$ tests, respectively. The Wilcoxon test will be used to analyse the statistical significance of the pre- and post-intervention variables. The above non-parametric tests will be used, if the data either do not conform to normality or cannot be normalised by log-transformation. Otherwise, an analysis of covariance will be used to compare the differences between two groups with the baseline data as the covariate and the potential confounders added to the model. Contingency tables will be used to assess relationships between the categorical variables. Depending on the data distribution, parametric (Pearson's) or nonparametric tests (Spearman's) will be applied to assess correlations. Uni- and multivariate logistic and linear regression analyses will be used to identify independent determinants of cognitive functions. Potentially confounding factors from these univariate analyses will subsequently be entered in a multivariate linear regression analysis. In a stepwise multivariate analysis, factors 
for inclusion will be set at $p<0.1$. In terms of the categorical variables, dummy variables should be entered in the linear regression analysis. If any data are missing, we will assume that they all follow a multivariate normal distribution and adopt multiple imputation approaches. There are no planned interim statistical analyses, or formal stopping rules with regard to efficacy. For the main-outcome parameters, a correction for multiple testing will be applied, unless a multivariate model can be used which produces one single test.

\section{Measurable Effects}

This study will potentially provide additional information which allows a more efficient and precise planning of daily physical activity for $\mathrm{MCl}$ subjects. We expect that the study will produce exact values for the physical activity intensity required to protect against cognitive decline. The study findings might also be useful for developing first physical activity guidelines aiming to protect against cognitive impairment.

\section{Expected Results}

In the proposed randomised controlled trial, a 12-month physical activity intervention will be performed in a group of 198 subjects with $\mathrm{MCl}$ and aged $50-70$ years. On the basis of both the current literature and new findings, we aim to establish thresholds of the intensity and frequency of physical activity which will serve to develop novel physical activity guidelines to protect against cognitive decline in high-risk adults. We will also investigate associations between physical activity, cognitive function, brain volume, and blood biomarkers. We assume that there are thresholds of physical activity frequency and intensity, which improve global cognitive functions in at-risk individuals, preserving brain volumes and maintaining biomarker levels within the normal limits. The expected findings will allow us to develop the first specific, cognitive impairment-focused physical activity guidelines, which will be effective and achievable for older subjects. Walking, as a form of physical activity, is inexpensive, easy to perform and protects from other chronic diseases, such as diabetes, cardiovascular diseases, obesity, and depression. Therefore, a simple physical activity tracker with a mobile application could be a helpful tool in increasing compliance.

\section{Acknowledgements}

\section{Contributors}

M.J., A.M. and M.W.G. wrote the manuscript. J.B., H.W.W. and Z.P. commented on the manuscript, J.K.N., K.-H.H. and E.M. designed the study and edited the manuscript. J.W. designed the study and commented on the manuscript, as well as supervises and coordinates the study. All authors read and approved the final manuscript.

\section{Conflict of interest statement}

J.K.N. reports personal fees from Norsa Pharma, grant support from Biocodex Microbiota Foundation, and non-financial support from Nutricia, outside the submitted work. J.W. received personal fees and non-financial support from Biocodex, BGP Products, Chiesi, Hipp, Humana, Mead Johnson Nutrition, Merck Sharp \& Dohme, Nestle, Norsa Pharma, Nutricia, Roche, Sequoia Pharmaceuticals, and Vitis Pharma, as well as research grants, personal fees and non-financial support from Nutricia Research Foundation Poland, outside the submitted work. Other authors declare that they have no competing interests.

\section{Funding}

This research was funded by the National Science Centre (Twardowskiego Str. 16, 30-312 Kraków, Poland, https://ncn.gov.pl, telephone: +48 532082 239, fax: +48 1234190 99, e-mail: biuro@ncn.gov.pl), grant number UMO-2017/27/B/NZ7/02924. The sponsor and study participants had no role in the study design and will have no role in the collection, management, analysis, and interpretation of data, writing of the report, and the decision to submit the report for publication.

\section{References}

1. Petersen RC, Lopez O, Armstrong MJ, Getchius TSD, Ganguli M, Gloss D, Gronseth GS, Marson D, Pringsheim T, Day GS, Sager M, Stevens J, Rae-Grant A. Practice guideline update summary: Mild cognitive impairment: Report of the Guideline Development, Dissemination, and Implementation Subcommittee of the American Academy of Neurology. Neurology. 2018 Jan 16;90(3):126-135. doi: 10.1212/ WNL.0000000000004826.

2. Ward A, Tardiff S, Dye C, Arrighi HM. Rate of conversion from prodromal Alzheimer's disease to Alzheimer's dementia: a systematic review of the literature. Dement Geriatr Cogn Dis Extra. 2013 Sep 28;3(1):320-332. doi: 10.1159/000354370.

3. GBD 2016 Dementia Collaborators. Global, regional, and national burden of Alzheimer's disease and other dementias, 1990-2016: a systematic analysis for the Global Burden of Disease Study 2016. Lancet Neurol. 2019 Jan;18(1):88-106. doi: 10.1016/S14744422(18)30403-4.

4. World Health Organization. Risk reduction of cognitive decline and dementia. [cited 2020 Jan 29]. Available from: http://www.who.int/mental_health/neurology/dementia/guidelines_risk_reduction/en/

5. Kivipelto $M$, Ngandu T, Laatikainen T, Winblad B, Soininen $\mathrm{H}$, Tuomilehto J. Risk score for the prediction of dementia risk in 20 years among middle aged 
people: a longitudinal, population-based study. Lancet Neurol. 2006 Sep;5(9):735-741. doi: 10.1016/ S1474-4422(06)70537-3.

6. Karakaya T, Fußer F, Schröder J, Pantel J. Pharmacological treatment of mild cognitive impairment as a prodromal syndrome of Alzheimer's disease. Curr Neuropharmacol. 2013 Jan;11(1):102-108. doi: 10.2174/157015913804999487.

7. Northey JM, Cherbuin N, Pumpa KL, Smee DJ, Rattray B. Exercise interventions for cognitive function in adults older than 50: a systematic review with meta-analysis. Br J Sports Med. 2018 Feb;52(3):154160. doi: 10.1136/bjsports-2016-096587.

8. Smith PJ, Blumenthal JA, Hoffman BM, Cooper H, Strauman TA, Welsh-Bohmer K. Aerobic exercise and neurocognitive performance: a meta-analytic review of randomized controlled trials. Psychosom Med. 2010 Apr;72(3):239-252. doi: 10.1097/ PSY.0b013e3181d14633.

9. Erickson KI, Raji CA, Lopez OL, Becker JT, Rosano C, Newman AB, Gach HM, Thompson PM, Ho AJ, Kuller LH. Physical activity predicts gray matter volume in late adulthood: the Cardiovascular Health Study. Neurology. 2010 Oct 19;75(16):1415-1422. doi: 10.1212/WNL.0b013e3181f88359.

10. Ströhle A, Schmidt DK, Schultz F, Fricke N, Staden T, Hellweg R, Priller J, Rapp MA, Rieckmann $N$. Drug and exercise treatment of alzheimer disease and mild cognitive impairment: a systematic review and meta-analysis of effects on cognition in randomized controlled trials. Am J Geriatr Psychiatry. 2015;23(12):1234-1249. doi: 10.1016/j. jagp.2015.07.007.

11. Garber CE, Blissmer B, Deschenes MR, Franklin BA, Lamonte MJ, Lee IM, Nieman DC, Swain DP; American College of Sports Medicine. American College of Sports Medicine position stand. Quantity and quality of exercise for developing and maintaining cardiorespiratory, musculoskeletal, and neuromotor fitness in apparently healthy adults: guidance for prescribing exercise. Med Sci Sports Exerc. 2011 Jul;43(7):13341359. doi: 10.1249/MSS.0b013e318213fefb.

12. Troiano RP, Berrigan D, Dodd KW, Mâsse LC, Tilert $\mathrm{T}$, McDowell M. Physical activity in the United States measured by accelerometer. Med Sci Sports Exerc. 2008 Jan;40:181-188. doi: 10.1249/ mss.0b013e31815a51b3.

13. Chan AW, Tetzlaff JM, Altman DG, Laupacis A, Gøtzsche PC, Krleža-Jerić K, Hróbjartsson A, Mann $\mathrm{H}$, Dickersin K, Berlin JA, Doré CJ, Parulekar WR, Summerskill WS, Groves T, Schulz KF, Sox HC, Rockhold FW, Rennie D, Moher D. SPIRIT 2013 statement: defining standard protocol items for clinical trials. Ann Intern Med. 2013 Feb;158(3):200-207. doi: 10.7326/0003-4819-158-3-201302050-00583.
14. Chan AW, Tetzlaff JM, Gøtzsche PC, Altman DG, Mann $H$, Berlin JA, Dickersin K, Hróbjartsson A, Schulz KF, Parulekar WR, Krleza-Jeric K, Laupacis A, Moher D. SPIRIT 2013 explanation and elaboration: guidance for protocols of clinical trials. BMJ. 2013 Jan;346:e7586. doi: 10.1136/bmj.e7586.

15. Schulz KF, Altman DG, Moher D; CONSORT Group. CONSORT 2010 Statement: updated guidelines for reporting parallel group randomised trials. BMC Med. 2010 Mar;8:18. doi: 10.1186/1741-7015-8-18.

16. Zhu W, Wadley VG, Howard VJ, Hutto B, Blair SN, Hooker SP. Objectively measured physical activity and cognitive function in older adults. Med Sci Sports Exerc. 2017 Jan;49(1):47-53. doi: 10.1249/ MSS. 0000000000001079.

17. Clinical Research APPS. RRApp Robust Randomization App. [cited 2021 Jun 22]. Available from: http:// clinicalresearch-apps.com/RRApp.html

18. Stergiou GS, Palatini P, Parati G, O'Brien E, Januszewicz A, Lurbe E, Persu A, Mancia G, Kreutz R; European Society of Hypertension Council and the European Society of Hypertension Working Group on Blood Pressure Monitoring and Cardiovascular Variability. 2021 European Society of Hypertension practice guidelines for office and out-of-office blood pressure measurement. J Hypertens. 2021 Jul;39(7):12931302. doi: $10.1097 / \mathrm{HJH} .0000000000002843$.

19. Jeżewska-Zychowicz M, Gawęcki J, Wądołowska L, Czarnocińska J, Galiński G, Kołłajtis-Dołowy A, Roszkowski W, Wawrzyniak A, Przybyłowicz K, Krusińska B, Hawrysz I, Słowińska MA, Niedźwiedzka E. Kwestionariusz do badania poglądów i zwyczajów żywieniowych dla osób w wieku od 16 do 65 lat, wersja 1.2 [Beliefs and eating habits questionnaire for subjects aged 16 to 65, version 1.2.]. In: Gawęcki J, editor. Kwestionariusz do badania poglądów i zwyczajów żywieniowych oraz procedura opracowania danych [Beliefs and eating habits questionnaire and the data processing procedure]. Warszawa: Komitet Nauki o Żywieniu Człowieka Polskiej Akademii Nauk; 2014. p. 21-33.

20. Jarosz M, Rychlik E, Stoś K, Charzewska J. Normy żywienia dla populacji Polski i ich zastosowanie [Nutrition standards for the Polish population and their application]. Warszawa: Narodowy Instytut Zdrowia Publicznego - Państwowy Zakład Higieny; 2020.

21. Hamilton M. A rating scale for depression. J Neurol Neurosurg Psychiatry.1960;23(1):56-62. doi: 10.1136/ jnnp.23.1.56.

22. American Psychiatric Association. Handbook of psychiatric measures. Washington DC: American Psychiatric Association; 2000. 